\title{
A theorist's view of the A-star laboratory
}

\author{
Hiromoto Shibahashi \\ Department of Astronomy, University of Tokyo, Tokyo 113-0033, Japan \\ email: shibahashi@astron.s.u-tokyo.ac.jp
}

\begin{abstract}
Two topics concerning A-type stars are discussed: starspots associated with the strong magnetic fields and the prospects for asteroseismology. Considering starspots as analogous to sunspots is misleading. The photosphere of starspots of a magnetic A-star is higher than the normal photosphere, contrary to sunspots. As for the prospects of asteroseismology, it is demonstrated how well (or poorly) we can probe the internal structure of the distant stars using a limited number of p-mode frequencies. Although the detectable eigenmodes must be limited to be $\ell \leqslant 4$, if the observational error is of the order of $10^{-3}$, inversion has still some hope.
\end{abstract}

Keywords. Stars: chemically peculiar, stars: interiors, stars: oscillations, stars: spots

\section{Is the level of starspots of Ap stars lower than its surroundings?}

\subsection{Introduction: Gough's argument}

Many Ap stars are known to have strong magnetic fields, which seem to be, roughly speaking, approximately dipole fields. In most cases the magnetic field strength is cyclically varying, and such variation is thought to be caused by the rotation of the star whose magnetic axis is inclined to the rotation axis. The photometric changes are dependent on the color band used in the observations. Typically the star is darkest in the $V$-band at the phase of magnetic maximum. The correlation between the darkness and the magnetism reminds us of sunspots. We believe that the Ap stars have global dipole-like magnetic fields, of the order of several hundreds Gauss or more, associated with giant starspots, although the mechanism generating such starspots and the nature of their structure have not been established.

The geometrical level of sunspots is slightly lower than the surrounding photospheric level. This can be seen when we see sunspots near the limb. One might think that the situation is similar for the starspots of Ap stars. Considering starspots as the analogs of sunspots is, however, misleading. We need to pay attention to the differences between starspots and sunspots. Gough (2003) pointed out that the starspots of Ap stars must be higher than their surroundings. Starspots are not transient like sunspots and their size is very large, occupying perhaps $20 \%$ or more of a hemisphere. With this in mind, he and his colleagues modelled the equilibrium envelope in the magnetic polar regions by suppressing convection and using a normal envelope in the magnetic equatorial zone (Balmforth et al. 2001). The outcome of their calculation was that the starspots' photosphere is only slightly cooler than the normal photosphere and that the starspots' photosphere is slightly higher than the normal photosphere. Since this conclusion might be contrary to what one might expect, we carry out a 'Gedankenexperiment' to see if the level of starspots is indeed higher than the normal photosphere.

\subsection{Radiative case}

Let us consider a radiative envelope. For early A-type stars, the convection zones associated with the hydrogen and the helium ionization zones are thin and shallow. Convective 
energy transport is inefficient so that the envelope is almost radiative. It is expected that, due to the magnetic pressure, the gas pressure in the magnetic polar regions is lower than in the normal envelope. A decrease in gas pressure would lead to a decrease in temperature, if the density were unchanged. However, the magnetic polar regions may be quite large. Hence the thermal balance on a global scale should be taken into account. A decrease in temperature would lead to a steep temperature gradient, if the radius were kept unchanged. But the temperature gradient is determined by the amount of radiation flux:

$$
\boldsymbol{F}_{\mathrm{rad}}=-\frac{4 a c}{3 \kappa \rho} T^{3} \nabla T,
$$

where $\boldsymbol{F}_{\text {rad }}$ denotes the radiative energy flux, $T$ the temperature, $\kappa$ the opacity, $\rho$ the density, $a$ the radiation density constant, $c$ the speed of the light, and $\nabla$ the gradient operator. To maintain the temperature gradient, the magnetic polar regions have to expand slightly. Consequencely the density becomes lower there. A decrease in gas pressure is mainly compensated by a decrease in density rather than in temperature, and the level of the photosphere of the magnetic polar regions is slightly higher than the normal photosphere. The magnetic polar regions must look like a pair of big plateaus.

\subsection{Convective case}

For stars such as F-type or late A-type stars, with smaller effective temperatures, the efficiency of convective energy transport becomes more important. Magnetic fields work to suppress convection or to lower the efficiency of convective energy transport. In a uniform sector of a magnetic field, whether the motion in the layer is monotonically growing (dynamically unstable) is dependent on the superadiabaticity and the Alfvén frequency. The latter is dependent on the wavenumber $\boldsymbol{k}$ and the magnetic field $\boldsymbol{B}$. For a dipole field, the magnetic field is almost vertical in the magnetic polar regions and almost horizontal near the magnetic equator of the star. Since $\boldsymbol{B} \cdot \boldsymbol{k}$ is large in the magnetic polar regions, the monotonic convection motion may be suppressed and the motion may be oscillatory there. On the other hand, the motion is supposed to be convectively unstable at the magnetic equator of the star, because $\boldsymbol{B} \cdot \boldsymbol{k}$ is small there.

In the magnetic polar regions, suppression of the convective energy transport efficiency leads to a steep temperature gradient. If the radius of the star were unchanged, the temperature there would become cooler. This means that the total luminosity, $L=$ $\pi R^{2} \sigma T_{\text {eff }}^{4}$, would decrease, where $\sigma$ and $T_{\text {eff }}$ denote the Stefan-Boltzmann constant and the effective temperature, respectively. If we assume that the star is in thermal balance, the luminosity of the star is determined by the nuclear energy generation, which is not influenced by the surface magnetic fields, and should be unchanged. To keep the total luminosity unchanged, the stellar radius has to be slightly larger. Thus the elevation of the photosphere in the magnetic polar regions must be slightly higher than in the equatorial region. The magnetic polar regions are a pair of slightly cool plateaus.

\subsection{Summary and discussion}

In both cases, we have reached the same conclusion as Gough (2003). The geometric level of the photosphere of starspots is higher than its surroundings, contrary to the case of sunspots. Starspots in the magnetic polar regions should be regarded as a pair of slightly cool plateaus with a slightly lower density. For an Ap star with $T_{\text {eff }} \simeq 8000 \mathrm{~K}$ and $B \simeq 1 \mathrm{kG}$, the temperature difference between the photosphere of the magnetic polar regions (starspots) and the that of the magnetic equator is estimated to be of the order of several hundreds Kelvin. Since the temperature of the magnetic polar regions is expected to be lower than the magnetic equator of the star, some energy transfer is expected to 
occur from the magnetic equator to the poles. This might cause chemical diffusion in the horizontal direction. The lower density at the magnetic polar regions enhances the pulsation amplitude there compared to the magnetic equator.

\section{Prospects of asteroseismology: how much information can we get from asteroseismology?}

\subsection{Introduction}

Stimulated by the success of helioseismology, a similar attempt to probe the internal structure of stars in general has been encouraged. Uninterrupted, long-term observations with high precision photometry or Doppler measurements from space have been proposed. MOST is indeed now working (Matthews et al. 2004). A-type stars should be among the target stars of asteroseismology. Many $\delta$ Scuti stars are rich in observed oscillation frequencies. roAp stars are unique objects as they have very strong magnetism and chemical peculiarity. However, the seismological approach to stars in general is much more difficult than that for the Sun. The great success of helioseismology is based on: (1) the large number of oscillation modes that have been detected, (2) the modes have been well identified, and (3) a good solar model is available which can be used as a reference model for the inversion procedure. The situation is different for distant stars. The stellar image usually cannot be resolved into a two-dimensional disk image. For a star oscillating with a high $\ell$ mode, the stellar surface is divided into many small regions oscillating in different phases, and then the contributions of each region are canceled by others so that the total amplitude of the variability of the star is too small to be detected. As a consequence, the observable eigenmodes in an individual star are likely to be restricted to only those with $\ell \leqslant 4$. In this situation, how much information can we get by asteroseismology about the invisible interior of stars? What is the limit?

\subsection{Numerical experiments}

Now by assuming that the mode identification is complete and that a good model is available, I demonstrate how well we can probe the invisible interior of stars by utilizing a limited number of oscillation modes. The principal aim is to find the limit of seismic inversion in the best case. We can use simple stellar models suitable for this purpose, otherwise we would have to worry about various uncertainties in the physics. Let us adopt two polytrope stars, one with the polytropic index $N=3.01$ and the other with $N=3.00$. We treat the calculated eigenfrequencies of the polytrope $N=3.01$ as if they were the observational data, and try to reproduce the model by carrying out the inversion of this data set. The polytrope star with $N=3.00$ is adopted as a reference model in the inversion procedure. Since the equation to solve in constructing models is the well-known Lane-Emden equation (see, e.g., Hansen \& Kawaler 1994), anyone can reproduce the following results. However, we should be careful about the numerical precision. Eigenfrequencies of linear, adiabatic, radial and nonradial p-modes are computed by solving the pulsation equations in the same manner, that is, the four first-order differential equations are solved even in the case of $\ell=0$ (cf. Unno et al. 1989). Since the models are polytropic stars, the mechanical outer boundary condition for pulsation is the zero boundary condition, that is, the Lagrangian perturbation of pressure is set to be zero, $\delta p=0$, at the surface.

The p-mode pulsation characteristics of a star are determined by the sound-speed and the density profiles in the star. It is known that the eigenfrequencies obey a variational principle. Hence, if we take the polytrope star with $N=3.00$ as a reference model and 

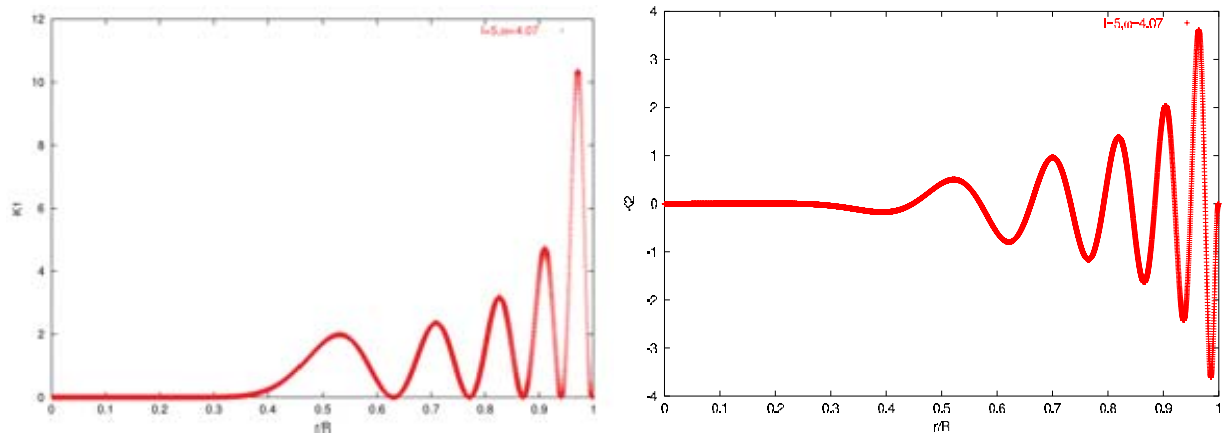

Figure 1. Examples of the kernels $K_{n, \ell}^{(1)}(r)$ (left panel) and $K_{n, \ell}^{(2)}(r)$ (right panel) of the polytrope star with $N=3.0$. These are the kernels for p $_{5}$-mode of $\ell=5$.
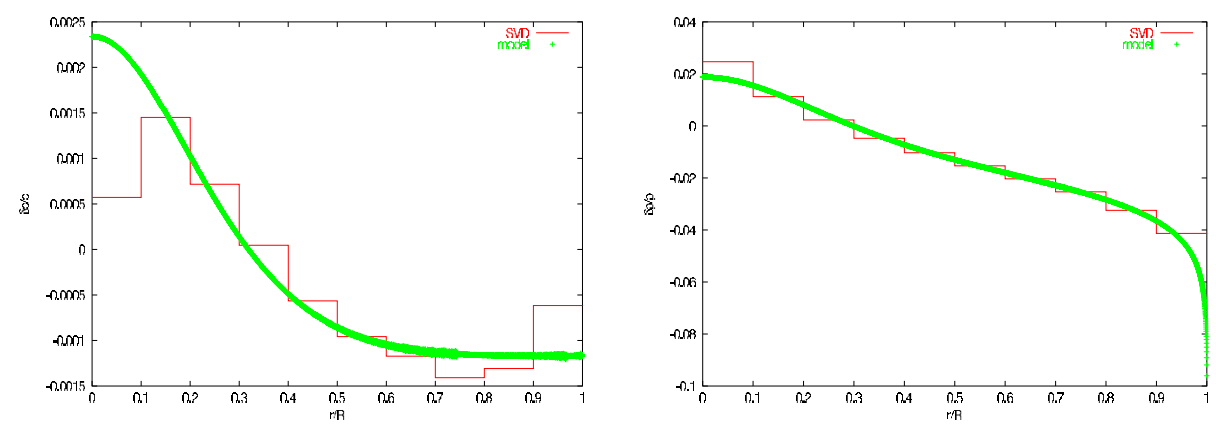

Figure 2. The results of inversion of p-modes with $\ell \leqslant 100$ and $\omega^{2} /\left(G M / R^{3}\right) \leqslant 2000$. We assumed in the inversion procedure that each of the differences is a piecewise constant function with a step of $\Delta r / R=0.1$. The left panel shows the inferred difference in the sound-speed profile and the true difference (a smoothed curve), while the right panel shows that in the density profile.

compare its eigenfrequencies with those of the polytrope star $N=3.01$, the difference in the eigenfrequencies between them are expressed in terms of the differences in the sound-speed and the density profiles between these two stars:

$$
\frac{\delta \omega_{n, \ell}}{\omega_{n, \ell}}=\int\left\{\frac{\delta c}{c} K_{n, \ell}^{(1)}(r)+\frac{\delta \rho}{\rho} K_{n, \ell}^{(2)}(r)\right\} d r .
$$

where $\omega_{n, \ell}$ and $\boldsymbol{\xi}_{n, \ell}$ denote the eigenfrequency and the normalized eigenfunction of the mode $\left(\omega_{n, \ell}^{2} \int \boldsymbol{\xi}_{n, \ell}^{*} \cdot \boldsymbol{\xi}_{n, \ell} d m=1\right)$ with radial order $n$ and spherical degree $\ell$, respectively, and $c(r)$ and $\rho(r)$ denote the sound-speed and the density profiles, respectively, as functions of the distance $r$ to the center. Equation (2.1) should be regarded as a set of integral equations with two unknown functions $\delta c(r)$ and $\delta \rho(r)$, and $K_{n, \ell}^{(1)}(r)$ and $K_{n, \ell}^{(2)}(r)$ are the kernels which are computed with the reference model for the given mode. The explicit expression of the kernels $K_{n, \ell}^{(1)}(r)$ and $K_{n, \ell}^{(2)}(r)$ can be seen in Gough \& Thompson (1991). Figure 1 shows the kernels for $\mathrm{p}_{5}$ mode of $\ell=5$. Various sophisticated methods of solving this type of integral equations have been invented. Here, we solve a set of equations (2.1) for various eigenmodes with the help of the singular value decomposition (SVD) and obtain the unknown functions $\delta c(r)$ and $\delta \rho(r)$ (cf. Press et al. 1992).

Let us see first that, if modes up to $\ell=100$ were detected, the true difference of the star would be admirably well reproduced from the reference model. For this purpose, let 

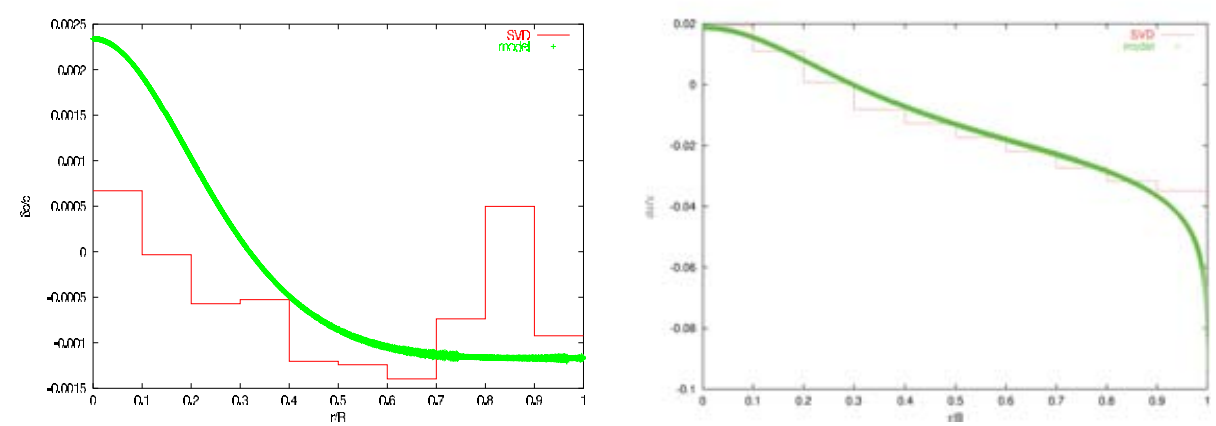

Figure 3. The results of inversion of p-modes with $\ell<5$ and $\omega^{2} /\left(G M / R^{3}\right) \leqslant 2000$. In the inversion procedure each of the differences is assumed to be a piecewise constant function with a step of $\Delta r / R=0.1$. The left panel shows the inferred difference in the sound-speed profile and the true difference (a smoothed curve), while the right panel shows that in the density profile.

us use all the p-modes with $\ell \leqslant 100$ and $\omega^{2} /\left(G M / R^{3}\right) \leqslant 2000$. The total number of modes used in the inversion is then 450 . Here we assume that both $\delta c(r)$ and $\delta \rho(r)$ are piecewise constant functions with a step of $\Delta r / R=0.1$, rather than smooth function, that is, we consider an expansion of $\delta c(r)$ and $\delta \rho(r)$ in terms of a piecewise constant function. The problem is then to determine the twenty coefficients for the piecewise constant functions from 450 equations. Figure 2 shows the inverted results. In each panel, the true difference between the star (the polytrope star with $N=3.01$ ) and the reference model (the polytrope star with $N=3.0$ ) is shown as a smooth function. As seen in this figure (particularly in the left panel showing $\delta c / c$ ), the inversion reproduces admirably well the true difference, particularly in the range of $0.2 \leqslant r / R \leqslant 0.9$. The gap between the inverted results and the true difference is substantial for $r / R \leqslant 0.1$. This is partly because the number of modes reaching near the center is small and partly because the sound speed is so high near the center that the sound wave is insensitive to this region. The gap between the inverted results and the true difference is also substantial for $0.9 \leqslant r / R$. This is because the number of modes for which the inner turning point is located near the surface is small. These tendency is seen in the helioseismic inversion.

Let us reduce the range of the spherical degree $\ell$ of eigenmodes to $0 \leqslant \ell \leqslant 4$. However, since the current aim is to prospect the ultimately reachable possibility of asteroseismology in the extremely idealistic case, let us still adopt all the p-modes for which frequency $\omega^{2} /\left(G M / R^{3}\right) \leqslant 2000$, using a total of 200 p-modes in the inversion. The results are shown in Fig. 3. As seen in this figure, the SVD inversion whose results are shown by the step functions is much worse than the case of inversion of modes with $\ell \leqslant 100$, but it is still satisfactorily successful in such an idealistic case. The total number of modes has been obviously overestimated, and the mode identification has been assumed to be perfect. Furthermore, it has been assumed that the observational frequency determination is error free. Obviously these assumptions are unrealistic. But we should regard these inverted results as the ultimately reachable points of asteroseismology. We cannot expect better results than these, but we can expect these results in the extremely idealistic case.

Since the frequency data are assumed to be error free, all the inversion error is the systematic error, that is, the intrinsic error which is the dependent on the inversion method (SVD in the present case). One might get an impression from Fig. 3 that the density profile is reproduced better than the sound-speed profile. This is not true. The left panel of Fig. 4 shows the systematic error of the inverted results shown in Fig. 3. It clearly shows that the systematic error in the sound-speed inversion is smaller than that 

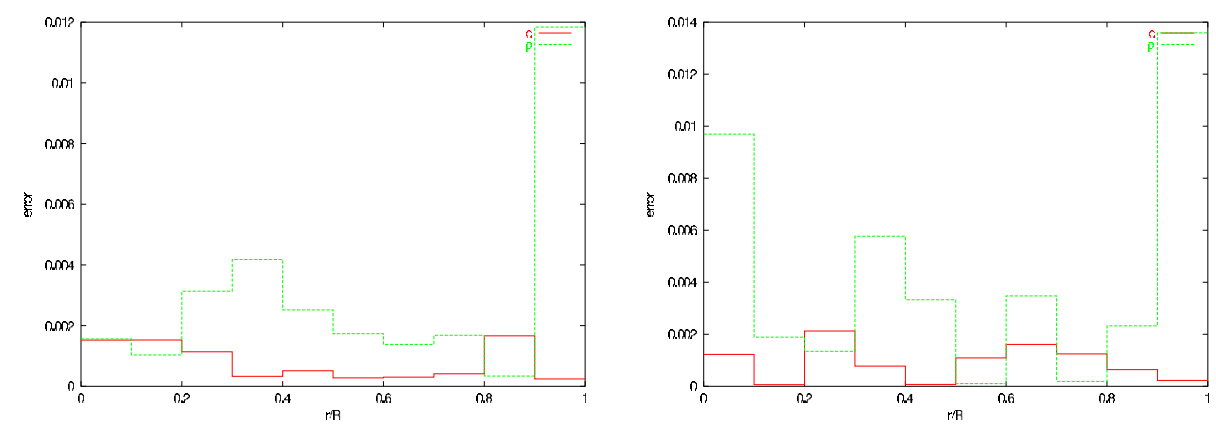

Figure 4. Left: Systematic error (intrinsic error which is dependent on the inversion method; SVD in the present case): It should be noted that systematic error in the sound-speed inversion is smaller than that in the density inversion. Right: Total error in the case that observational error is $0.03 \%$.

in the density inversion. In reality, observational error is unavoidable. As a simulation, let us add $0.03 \%$ error into the observational data set and estimate its effect on inversion. An observational error of $0.03 \%$ can be reached with 3 -months of uninterrupted photometric observations, if the period is measured with accuracy of $10 \mathrm{~s}$ for a single cycle of pulsation in the case where the period is $300 \mathrm{~s}$. The right panel of Fig. 4 shows the total error in this case. We would say that inversion still has some hope.

\subsection{Summary and discussion}

We demonstrated how well (or poorly) we can probe the internal structure of the distant stars using a limited number of p-mode frequencies. Although the detectable eigenmodes must be limited to be $\ell \leqslant 4$, the inversion is still highly satisfactory in the extremely idealistic (200 modes with no observational error) case. We conclude from the present simulation that if the observational error is of the order of $10^{-3}$, inversion still has some hope.

We restricted ourselves to inversion based on the absolute values of frequencies. However, there are other possibilities of inversion based on the relative differences among the eigenfrequencies, such as the frequency difference between the consecutive p-modes for which radial order $n$ differs by 1 , or the frequency difference between the p-modes for which the radial orders are the same but their spherical degrees $\ell$ differ by 2 . Another example is the second derivative, $\omega_{n+1, \ell}-2 \omega_{n, \ell}+\omega_{n-1, \ell}$, which is sensitive to a discontinuity in the stellar structure.

\section{References}

Balmforth, N.J., Cunha, M.S., Dolez, N., Gough, D.O., Vauclair, S. 2001, A $\& A$, 323, 362

Gough, D. 2003, in: L.A. Balona, H.F. Henrichs \& R. Medupe (eds.), Magnetic fields in $O, B$ and A stars: Origin and connection to pulsation, rotation and mass loss, ASP Conf. Ser. (San Francisco: Astron. Soc. Pacific), vol. 305, p. 389

Gough, D.O. \& Thompson, M.J. 1991, in: A.N. Cox, W.C. Livingston \& M.S. Matthews (eds.), Solar Interior and Atmosphere (The University of Arizona Press), p. 519

Hansen, C.J. \& Kawaler, S.D. 1994, Stellar Interiors (Springer)

Matthews, J.M., Kuschnig, R., Guenther, D.B., Walker, G.A.H., Moffat, A.F.J., Rucinski, S.M., Sasselov, D., Weiss, W.W. 2004, Nature, 430, 51

Press, W.H., Teukolsky, S.A., Vetterling, W.T., Flannery, B.P. 1992, Numerical Recipes in Fortran (Second Edition) (Cambridge University Press) 
Unno, W., Osaki, Y., Ando, H., Saio, H., Shibahashi, H. 1989, Nonradial Oscillations of Stars (Second Edition) (University of Tokyo Press)

\section{Discussion}

BALONA: The eigenfrequencies of a roAp star are affected by the magnetic field. Therefore we need to correct the observed frequencies for this effect before the data can be used for asteroseismology. I believe we are still very far from being able to do this with confidence, so maybe roAp stars are, at present, not good candidates for asteroseismology.

SHIBAHASHI: Correcting the magnetic effect for the frequencies is hard. The inversion of the absolute values of the observed frequencies may be inappropriate for roAp stars at present as you point out. Probably taking the differences or the differentiation of the observed frequencies may be useful in this case.

BREGER: I would like to be more optimistic. For normal A stars we can easily detect $\ell>5$ modes through line-profile changes. The $\ell=|m|=8 \sim 14$ modes are easily detected. With better observations to account for narrower lines and short periods $\ell \geqslant 5$ could (or might) be doable.

Michel: I also have a more optimistic point of view. You adopted a polytrope model for simplicity and clarity of the demonstration, but this type of model omits considerable physics which we are strongly interested in and that we want to probe with stellar seismology. The existence of a convective core and of a steep $\mu$-gradient at its edge is one example. Models show that the existence of mixed modes in stars more massive than the Sun makes possible the building of inversion kernels sensitive to this very deep region, while the center of the Sun is still out of reach because of the lack of observed g-modes. Another example would be the depth of the outer convection zone which can be probed with pseudo-oscillations in the second order difference, for instance, with the same efficiency using low $\ell$ modes only or high $\ell$ modes. I could also quote published papers showing that for $\delta$ Scuti stars, for instance, there is great hope to obtain by inversion the rotational profile, using only low $\ell$ modes (Goupil, M.-J. et al. 1996, A\&A $305,487)$.

ShiBAhashi: Yes, taking the differences or the differentiation of the observed frequencies opens another possibility of inversion in asteroseismology. What I intended to stress here is that even a limited number of modes, much smaller than the solar case, are potentially very useful to deduce detailed information on the structure of the star.

Cunha: In Balmforth et al. (2001) we modelled the star by a spot, where convection is suppressed, and the equatorial region, where it is not suppressed. To keep the interior the same, we have allowed for a change its effective temperature, $T_{\text {eff }}$, and the luminosity $L$ at the surface. The luminosity $L$ was allowed to change to account for a 'side-leak' of energy, due to the difference in energy transport in the two regions. Can you please comment on the differences between this spot model and the model you described?

H. SHIBAHASHI: If a 'side-leak' is allowed, the temperature of starspots must be lower than the case of no side-leak, otherwise the total luminosity of the star would be larger than the energy generation. I think the hydrostatic balance is handled well in Balmforth et al. (2001), but I am not certain that the thermal balance is handled well in their model. 